\title{
Remdesivir-induced symmetrical drug-related intertriginous and flexural exanthema (SDRIFE)? A case report with review of the literature
}

\author{
Johannes Heck ${ }^{1}$ (D) Dirk O. Stichtenoth ${ }^{1} \cdot$ Roland Mettin $^{2}$ (D) Jörg Jöckel ${ }^{2} \cdot$ Christoph Bickel $^{2}$. \\ Benjamin Krichevsky ${ }^{2}$ (D)
}

Received: 11 June 2020 / Accepted: 3 July 2020 / Published online: 6 August 2020

(C) The Author(s) 2020

Remdesivir is an antiviral agent which is under clinical investigation for the treatment of coronavirus disease 2019 (COVID-19) [1]. Besides hypotension, deep-vein thrombosis, and delirium, rash has been reported as adverse drug reaction (ADR) of remdesivir [2]. The term "rash", although commonly applied in clinical trials, is too unspecific to allow for a differentiated cutaneous ADR (cADR) management.

Symmetrical drug-related intertriginous and flexural exanthema (SDRIFE) is an immune-mediated cADR which occurs after systemic administration of a drug in an individual without prior sensitization [3, 4]. SDRIFE is clinically defined by five criteria: (i) onset after initial or repeated exposure to a systemically administered drug (contact allergens excluded), (ii) sharply demarcated erythema in the gluteal/perianal area and/or V-shaped erythema in the inguinal/perigenital region, (iii) involvement of at least one other intertriginous/flexural fold (e.g., axillae, antecubital fossae), (iv) symmetrical distribution, and (v) absence of systemic involvement [4]. Beta-lactam antibiotics, especially amoxicillin, are recognized as the most frequent trigger of SDRIFE $[4,5]$. The clinical course of SDRIFE usually is benign and self-limited. SDRIFE may, however, progress to a generalized maculopapular exanthema if the eliciting drug is not withdrawn [6]. Treatment of SDRIFE consists of discontinuation of

Electronic supplementary material The online version of this article (https://doi.org/10.1007/s00228-020-02955-4) contains supplementary material, which is available to authorized users.

Benjamin Krichevsky

BenjaminKrichevsky@bundeswehr.org

1 Institute for Clinical Pharmacology, Hannover Medical School, Hannover, Germany

2 Central Hospital of the German Armed Forces (Bundeswehrzentralkrankenhaus), Rübenacher Straße 170, 56072 Koblenz, Germany the offending agent. Additionally, antihistamines and topical/ systemic glucocorticosteroids may provide symptomatic relief and speed up the healing process $[5,7,8]$.

A 67-year-old male patient suffering from COVID-19 was transferred from Northern Italy to the Central Hospital of the German Armed Forces (Bundeswehrzentralkrankenhaus) Koblenz, Germany, on March 29, 2020, as part of an international medical evacuation operation. The patient had been mechanically ventilated since March 19 due to acute respiratory distress syndrome (ARDS). In Italy, the patient had been treated with intravenous remdesivir on a compassionate-use basis for four consecutive days (March 26 to 29). On March 30, the patient developed an erythematous macular exanthema on his trunk with pronounced involvement of both axillae, the inguinal and submental region. Remarkably, serous bullae with a diameter of up to $6 \mathrm{~cm}$ emerged in his axillae. The patient was physically examined by a senior dermatologist who established the diagnosis SDRIFE. Besides remdesivir, the patient was or had been treated with sufentanil, propofol, norepinephrine, pantoprazole, macrogol, metoclopramide, and enoxaparin. To evaluate the likelihood of each drug with regard to SDRIFE, we applied the Naranjo ADR Probability Scale (scale ranging from -4 to +13 , with higher scores indicating a higher probability) [9]. The score for remdesivir was +3 and for sufentanil, propofol, norepinephrine, pantoprazole, macrogol, metoclopramide, and enoxaparin was +1 , respectively, suggesting remdesivir as the primary offending agent. For a comprehensive medication chart of our patient as well as a detailed calculation of the score of each administered drug on the Naranjo ADR Probability Scale, please refer to Supplementary Table 1. Because of the concomitant viral ARDS, we refrained from glucocorticosteroid application. SDRIFE resolved spontaneously over the following weeks without further intervention. 
Table 1 Reported causative agents of symmetrical drug-related intertriginous and flexural exanthema (SDRIFE)

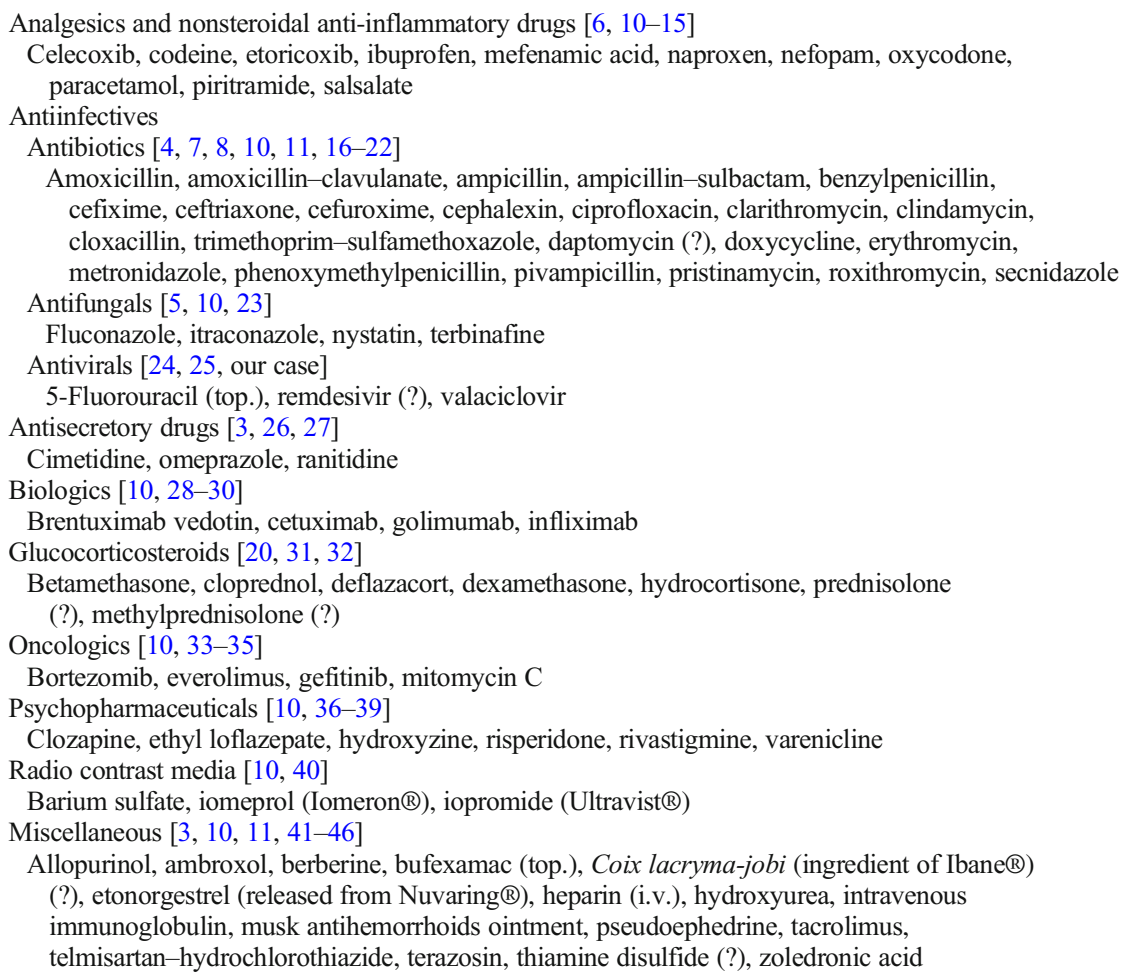

(?), possible association with SDRIFE; i.v., intravenous; SDRIFE, symmetrical drug-related intertriginous and flexural

exanthema; top., topical
PubMed was searched for case reports/series of SDRIFE in order to update preexisting lists of SDRIFE-eliciting drugs [3-5, $10,11]$. All identified publications were reviewed, and SDRIFEeliciting drugs were extracted. We provide a comprehensive summary of currently known triggers of SDRIFE (Table 1).

Depending on the clinical context, the differential diagnosis of SDRIFE includes other cADRs such as acute generalized exanthematous pustulosis, drug reaction with eosinophilia and systemic symptoms, and (multifocal) fixed drug eruption, as well as nondrug-related dermatologic conditions, e.g., tinea cruris and inverse psoriasis $[5,7,11]$. A plethora of cutaneous manifestations have been described in connection with COVID-19 [47], including an SDRIFE-like exanthema [48]. However, the clear temporal relationship between the start of treatment with remdesivir and the development of the exanthema as well as the improvement of the exanthema upon cessation of remdesivir lead us to the conclusion that our patient experienced an SDRIFE elicited by remdesivir. Nevertheless, we cannot rule out that the comedication contributed to the development of SDRIFE in the presented case.

Acknowledgments Open Access funding provided by Projekt DEAL. Johannes Heck and Benjamin Krichevsky dedicate this article to their common alma mater, the Julius Maximilian University (Julius-
Maximilians-Universität) Würzburg, Free State of Bavaria, Germany as well as to all their mentors during the study of human medicine.

Authors' contributions The first draft of the manuscript was written by Johannes Heck and Benjamin Krichevsky, and all authors commented on previous versions of the manuscript. All authors read and approved the final manuscript. This work was supervised by Dirk O. Stichtenoth.

\section{Compliance with ethical standards}

Conflict of interest The authors declare that they have no conflict of interest.

Consent for publication Informed consent for publication was obtained from the patient's legal guardian.

Open Access This article is licensed under a Creative Commons Attribution 4.0 International License, which permits use, sharing, adaptation, distribution and reproduction in any medium or format, as long as you give appropriate credit to the original author(s) and the source, provide a link to the Creative Commons licence, and indicate if changes were made. The images or other third party material in this article are included in the article's Creative Commons licence, unless indicated otherwise in a credit line to the material. If material is not included in the article's Creative Commons licence and your intended use is not permitted by statutory regulation or exceeds the permitted use, you will need to obtain permission directly from the copyright holder. To view a copy of this licence, visit http://creativecommons.org/licenses/by/4.0/. 


\section{References}

1. Beigel JH, Tomashek KM, Dodd LE, Mehta AK, Zingman BS, Kalil AC, Hohmann E, Chu HY, Luetkemeyer A, Kline S, Lopez de Castilla D, Finberg RW, Dierberg K, Tapson V, Hsieh L, Patterson TF, Paredes R, Sweeney DA, Short WR, Touloumi G, Lye DC, Ohmagari N, Oh MD, Ruiz-Palacios GM, Benfield T, Fätkenheuer G, Kortepeter MG, Atmar RL, Creech CB, Lundgren J, Babiker AG, Pett S, Neaton JD, Burgess TH, Bonnett T, Green M, Makowski M, Osinusi A, Nayak S, Lane HC (2020) Remdesivir for the treatment of Covid-19 - preliminary report. N Engl J Med. https://doi.org/10.1056/NEJMoa2007764

2. Grein J, Ohmagari N, Shin D, Diaz G, Asperges E, Castagna A, Feldt T, Green G, Green ML, Lescure FX, Nicastri E, Oda R, Yo K, QuirosRoldan E, Studemeister A, Redinski J, Ahmed S, Bernett J, Chelliah D, Chen D, Chihara S, Cohen SH, Cunningham J, D'Arminio Monforte A, Ismail S, Kato H, Lapadula G, L'Her E, Maeno T, Majumder S, Massari M, Mora-Rillo M, Mutoh Y, Nguyen D, Verweij E, Zoufaly A, Osinusi AO, DeZure A, Zhao Y, Zhong L, Chokkalingam A, Elboudwarej E, Telep L, Timbs L, Henne I, Sellers S, Cao H, Tan SK, Winterbourne L, Desai P, Mera R, Gaggar A, Myers RP, Brainard DM, Childs R, Flanigan T (2020) Compassionate use of remdesivir for patients with severe Covid-19. N Engl J Med 382: 2327-2336. https://doi.org/10.1056/NEJMoa2007016

3. Winnicki M, Shear NH (2011) A systematic approach to systemic contact dermatitis and symmetric drug-related intertriginous and flexural exanthema (SDRIFE): a closer look at these conditions and an approach to intertriginous eruptions. Am J Clin Dermatol 12:171-180

4. Häusermann P, Harr T, Bircher AJ (2004) Baboon syndrome resulting from systemic drugs: is there strife between SDRIFE and allergic contact dermatitis syndrome? Contact Dermatitis 51:297-310

5. Kumar S, Bhale G, Brar BK (2019) Symmetrical drug related intertriginous and flexural exanthema (SDRIFE) induced by fluconazole: an uncommon side effect of a commonly used drug. Dermatol Ther 32:e13130

6. Weiss D, Kinaciyan T (2018) Symmetrical drug-related intertriginous and flexural exanthema (SDRIFE) induced by mefenamic acid. JAAD Case Rep 5:89-90

7. Li DG, Thomas C, Weintraub GS, Mostaghimi A (2017) Symmetrical drug-related intertriginous and flexural exanthema induced by doxycycline. Cureus 9:e1836

8. Moreira C, Cruz MJ, Cunha AP, Azevedo F (2017) Symmetrical drug-related intertriginous and flexural exanthema induced by clarithromycin. An Bras Dermatol 92:587-588

9. Naranjo CA, Busto U, Sellers EM, Sandor P, Ruiz I, Roberts EA, Janecek E, Domecq C, Greenblatt DJ (1981) A method for estimating the probability of adverse drug reactions. Clin Pharmacol Ther 30:239-245

10. Magnolo N, Metze D, Ständer S (2017) Pustulobullous variant of SDRIFE (symmetrical drug-related intertriginous and flexural exanthema). J Dtsch Dermatol Ges 15:657-659

11. Nespoulous L, Matei I, Charissoux A, Bedane C, Assikar S (2018) Symmetrical drug-related intertriginous and flexural exanthema (SDRIFE) associated with pristinamycin, secnidazole, and nefopam, with a review of the literature. Contact Dermatitis 79: 378-380

12. Kim BJ, Kim HS, Lee JY, Kim HO, Park YM, La HO (2014) Symmetrical drug-related intertriginous and flexural exanthema caused by celecoxib. Int J Dermatol 53:1

13. Caralli ME, Seoane Rodriguez M, Rojas Perez-Ezquerra P, Pelta Fernandez R, De Barrio FM (2016) Symmetrical drug-related intertriginous and flexural exanthema (SDRIFE) caused by etoricoxib. J Investig Allergol Clin Immunol 26:128-129
14. Lugovic-Mihic L, Duvancic T, Vucic M, Situm M, Kolic M, Mihic J (2013) SDRIFE (baboon syndrome) due to paracetamol: case report. Acta Dermatovenerol Croat 21:113-117

15. Erfan G, Yanik ME, Kaya S, Tasolar K, Oznur M, Kulac M (2015) Symmetrical drug-related intertriginous and flexural exanthema due to codeine. Indian J Dermatol Venereol Leprol 81:405-406

16. Fischbach S, Hall N, Bains S, Selvaraj V (2018) An unusual case of worsening cellulitis: baboon syndrome or SDRIFE. BMJ Case Rep. https://doi.org/10.1136/bcr-2018-224503

17. Dogru M, Ozmen S, Ginis T, Duman H, Bostanci I (2012) Symmetrical drug-related intertriginous and flexural exanthema (baboon syndrome) induced by amoxicillin-clavulanate. Pediatr Dermatol 29:770-771

18. Sikar Akturk A, Bayramgurler D, Salman S, Yildiz KD, Odyakmaz Demirsoy E (2014) Symmetrical drug-related intertriginous and flexural exanthema (SDRIFE) induced by oral metronidazole. Cutan Ocul Toxicol 33:337-338

19. Cabrera Hernandez V, Gonzalez Afonso M, Callero Viera A, Martin-Fernandez Martin L (2019) Symmetrical drug-related intertriginous and flexural exanthema due to clindamycin. BMJ Case Rep 12:e230077. https://doi.org/10.1136/bcr-2019-230077

20. Treudler R, Simon JC (2006) Symmetric, drug-related, intertriginous, and flexural exanthema in a patient with polyvalent intolerance to corticosteroids. J Allergy Clin Immunol 118:965-967

21. Can C, Yazicioglu M, Ozdemir PG, Kilavuz S, Tastekin E (2014) Symmetrical drug-related intertriginous and flexural exanthema induced by two different antibiotics. Allergol Immunopathol (Madr) 42:173-175

22. Blackmur JP, Lammy S, Baring DE (2013) Baboon syndrome: an unusual complication arising from antibiotic treatment of tonsillitis and review of the literature. BMJ Case Rep 2013:bcr2013201977. https://doi.org/10.1136/bcr-2013-201977

23. Mohapatra M, Panda M, Kar BR, Raj C (2017) Symmetric drugrelated Intertriginous and flexural exanthema due to itraconazole: an uncommon side effect of a commonly used drug. Indian Dermatol Online J 8:501-503

24. Powers R, Gordon R, Roberts K, Kovach R (2012) Symmetrical drug-related intertriginous and flexural exanthema secondary to topical 5-fluorouracil. Cutis 89:225-228

25. Daito J, Hanada K, Katoh N, Katoh S, Sakamoto K, Asai J, Takenaka H, Kishimoto S (2009) Symmetrical drug-related intertriginous and flexural exanthema caused by valacyclovir. Dermatology 218:60-62

26. Kardaun SH, Tupker RA (2012) Symmetrical drug-related intertriginous and flexural exanthema (baboon syndrome) induced by omeprazole. Int J Dermatol 51:1134-1137

27. Binitha MP, Sasidharanpillai S, John R, Sherjeena PV (2014) Symmetrical drug-related intertriginous and flexural exanthema due to ranitidine. Indian J Pharm 46:551-552

28. Bulur I, Keseroglu HO, Saracoglu ZN, Gonul M (2015) Symmetrical drug-related intertriginous and flexural exanthema (Baboon syndrome) associated with infliximab. J Dermatol Case Rep 9:12-14

29. Elmariah SB, Cheung W, Wang N, Kamino H, Pomeranz MK (2009) Systemic drug-related intertriginous and flexural exanthema (SDRIFE). Dermatol Online J 15:3

30. Yang SY, Lan CC, Hu SC (2017) Symmetrical drug-related intertriginous and flexural exanthema (SDRIFE) induced by golimumab. Int J Dermatol 56:571-572

31. Garcia-Bravo B, Repiso JB, Camacho F (2000) Systemic contact dermatitis due to deflazacort. Contact Dermatitis 43:359-360

32. Armingaud P, Martin L, Wierzbicka E, Esteve E (2005) Baboon syndrome due to a polysensitization with corticosteroids. Ann Dermatol Venereol 132:675-677

33. Kurtzman DJ, Oulton J, Erickson C, Curiel-Lewandrowski C (2016) Everolimus-induced symmetrical drug-related 
Intertriginous and flexural exanthema (SDRIFE). Dermatitis 27: 76-77

34. Malissen N, Bourrain JL, Chiriac A, Montet A, Vincent L, Dereure O, du-Thanh A (2016) Symmetrical Intertriginous and flexural exanthema due to bortezomib (a proteasome inhibitor) given for myeloma. Acta Derm Venereol 96:995-996

35. Copps B, Lacroix JP, Sasseville D (2020) Symmetrical drug-related intertriginous and flexural exanthema secondary to epidermal growth factor receptor inhibitor gefitinib. JAAD Case Rep 6:172-175

36. Suvarna P, Kayarkatte MN, Shenoi SD, Jaiprakash P (2020) A rare case of clozapine-induced symmetrical drug-related intertriginous and flexural exanthema with vasculitis-like lesions. Contact Dermatitis 82:318-320

37. Allain-Veyrac G, Lebreton A, Collonnier C, Jolliet P (2011) First case of symmetric drug-related intertriginous and flexural exanthema (sdrife) due to rivastigmine? Am J Clin Dermatol 12:210-213

38. Akkari H, Belhadjali H, Youssef M, Mokni S, Zili J (2013) Baboon syndrome induced by hydroxyzine. Indian J Dermatol 58:244. https://doi.org/10.4103/0019-5154.110871

39. Cheng CY, Wang CW, Wang FY, Chung WH (2019) Vareniclineinduced symmetrical drug-related intertriginous and flexural exanthema. Indian J Dermatol Venereol Leprol 85:209-211

40. Arnold AW, Häusermann P, Bach S, Bircher AJ (2007) Recurrent flexural exanthema (SDRIFE or baboon syndrome) after administration of two different iodinated radio contrast media. Dermatology 214:89-93

41. Peeters D, Baeck M, Dewulf V, Tennstedt D, Dachelet C (2012) A case of SDRIFE induced by Nuvaring®. Contact Dermatitis 66: $110-111$

42. Cohen PR (2015) Zoledronic acid-associated symmetrical drugrelated intertriginous and flexural exanthema (SDRIFE): report of baboon syndrome in a woman with recurrent metastatic breast cancer after receiving zoledronic acid. Dermatol Online J 21:13030/ qt5kk0g864

43. Labadie JG, Florek AG, Croitoru A, Liu W, Krunic AL (2018) First case of symmetrical drug-related intertriginous and flexural exanthema (SDRIFE) due to Berberine, an over-the-counter herbal glycemic control agent. Int J Dermatol 57:e68-e70

44. Scherrer M, Araujo MG, Farah K (2018) Tacrolimus-induced symmetric drug-related intertriginous and flexural exanthema (SDRIFE). Contact Dermatitis 78:414-416

45. Hattori Y, Matsuyama K, Shu E, Kanoh H, Seishima M (2017) Symmetrical drug-related intertriginous and flexural exanthema possibly due to thiamine disulfide. J Dermatol 44:e115-e116

46. Chen B, Jiang X, Chen W, Qian T, Cheng H, Zhang D, Hao F (2020) A rare case of musk anti-hemorrhoids ointment-induced symmetrical drug-related intertriginous and flexural exanthema. Contact Dermatitis. https://doi.org/10.1111/cod.13574

47. Galvan Casas C, Catala A, Carretero Hernandez G, Rodriguez-Jimenez P, Fernandez Nieto D, Rodriguez-Villa Lario A et al (2020) Classification of the cutaneous manifestations of COVID-19: a rapid prospective nationwide consensus study in Spain with 375 cases. Br J Dermatol 183:71-77. https://doi.org/10.1111/bjd.19163

48. Mahe A, Birckel E, Krieger S, Merklen C, Bottlaender L (2020) A distinctive skin rash associated with coronavirus disease 2019? J Eur Acad Dermatol Venereol 34:e246-e247. https://doi.org/10. $1111 /$ jdv.16471

Publisher's note Springer Nature remains neutral with regard to jurisdictional claims in published maps and institutional affiliations. 